\title{
SYNTHESIS OF 1-(METHYLSULFONYL)-1-PROPENE AND N,N-DIMETHYL-1-PROPENE-1-SULFONAMIDE
}

\author{
I.D. Huzhvaa , E.H. Shvets ${ }^{b}$, M.A. Kolosov ${ }^{c}$ \\ V.N. Karazin Kharkiv National University, School of Chemistry, Svobody sq., 4, Kharkiv 61022, \\ Ukraine \\ a) $\square$ vanyaguzhva2016@gmail.com \\ b) $₫$ olena.h.shvets@karazin.ua \\ c)》kolosov@karazin.ua \\ https://orcid.org/0000-0002-3137-4553 \\ https://orcid.org/0000-0003-4791-2114 \\ https://orcid.org/0000-0002-6714-0513
}

Various vinylsulfones and vinylsulfonamides have a wide range of biological activities (mainly, inhibition of different types of enzymes) and are frequently used in synthetic organic chemistry (as active dienophiles, Michael acceptors and, generally, active agents in 1,4-addition and electrocyclization reactions). However, despite numerous synthesized substances of this type, the synthetic protocols for the obtaining of the low molecular weight representatives of these compounds - 1-(methylsulfonyl)-1-propene and $N, N$-dimethyl1-propene-1-sulfonamide - seem to be still little known. In the present work we report a simple, efficient and general protocol for the dehydrative synthesis of 1-(methylsulfonyl)-1-propene and $N, N$-dimethyl-1-propene1-sulfonamide starting from corresponding 1-(methylsulfonyl)-2-propanol and $N, N$-dimethyl2-hydroxypropanesulfonamide, respectively, using $\mathrm{MeSO}_{2} \mathrm{Cl} /$ organic base system basing on the preliminary experiment of 2-(4-bromophenyl)- $N, N$-dimethylethenesulfonamide synthesis from 2-(4-bromophenyl)2-hydroxy- $N, N$-dimethylethanesulfonamide. The latter in its turn has been obtained starting from $N, N$-dimethylmethanesulfonamide by lithiation with $n$-BuLi, subsequent action of 4-bromobenzaldehyde and further workup. The applied protocol of vinyl derivatives synthesis allows to avoid isolation of intermediate mesyl derivatives, consisting of one-pot formation of leaving group and its elimination. Accordingly to coupling constants in ${ }^{1} \mathrm{H}$ NMR spectra, synthesized $N, N$-dimethyl-1-propene-1-sulfonamide exists as mixture of $E$ - and Z-isomers (in the ratio 88:12), while isolated 1-(methylsulfonyl)-1-propene and 2-(4-bromophenyl)$N, N$-dimethylethenesulfonamide are the most stable $E$-isomers. The structures of the synthesized compounds are confirmed by the methods of ${ }^{1} \mathrm{H}$ NMR-spectroscopy and mass-spectrometry.

Keywords: vinylsulfones, vinylsulfonamides, 1-(methylsulfonyl)-1-propene, $N, N$-dimethyl-1-propene1-sulfonamide, dehydration.

\section{Introduction}

Vinylsulfones and vinylsulfonamides possess a variety of biological activities and this fact causes their wide application in medical chemistry [1-3]. Moreover, these compounds are active Michael acceptors, being intensively explored for the syntheses of $\beta$-functionalized sulfones and sulfonamides and in Diels-Alder reaction [4-7]

Nevertheless, the low molecular weight representatives of vinylsulfones and vinylsulfonamides $\mathrm{N}, \mathrm{N}$-dimethyl-1-propene-1-sulfonamide $\mathbf{1}$ and 1-(methylsulfonyl)-1-propene $\mathbf{2}$ - are still little known, and their synthetic preparation is not well-studied [8-10] (Figure 1).<smiles>CC=CS(=O)(=O)CC</smiles>

1<smiles>[Y16]C=CS(C)(=O)=O</smiles>

2

Figure 1. Structure of the target $N, N$-dimethyl-1-propene-1-sulfonamide 1 and 1-(methylsulfonyl)-1-propene 2.

The purpose of the present work is to work up simple and preparative protocols for the synthesis of compounds 1 and 2.

\section{Results and discussion}

First we tested ability of vinylsulfonamides' synthesis starting from corresponding $\beta$-hydroxy- $\mathrm{SO}_{2}$ derivatives using $\mathrm{MeSO}_{2} \mathrm{Cl} /$ organic base system on the example of transformation of bromoalcohol 3 
to corresponding vinylsulfonamide $\mathbf{4}$. Bromoderivatives $\mathbf{3}$ and $\mathbf{4}$ were chosen because of their relatively low solubility in most organic solvents (that was expected comparing with target compounds 1 and 2) and due to our ability to fix characteristic bromo-containing ions by MS-techniques.

Alcohol 3 was synthesized similarly to been reported [11, 12]. Initial $N, N$-dimethylmethanesulfonamide was metallated with $n$-BuLi in THF at the temperature below $0^{\circ} \mathrm{C}$ and formed salt was then treated with 4-bromobenzaldehyde in the same conditions, giving the product after appropriate workup (Scheme 1).<smiles>CS(C)(=O)=O</smiles>

1) $n$-BuLi (1.2 eq.), THF

2) 4-BrC $6 \mathrm{H}_{4} \mathrm{CHO}$ (1.1 eq.), THF

3) $\mathrm{NH}_{4} \mathrm{Cl}, \mathrm{H}_{2} \mathrm{O}$<smiles>CS(=O)(=O)CC(O)c1ccc(Br)cc1</smiles>

Scheme 1. Synthesis of alcohol 3.

Noteworthy, that all the preliminary experiments on the syntheses of tosylates and mesylates of $\beta$-hydroxy-SO ${ }_{2}$-derivatives 3, 5 and $\mathbf{6}$ anyway led (accordingly to ${ }^{1} \mathrm{H}$ NMR spectra of the probes of reaction mixtures) to formation of some amount of corresponding vinyl derivatives. That is why we undertook dehydration of secondary alcohol $\mathbf{3}$ according to the synthesis of the similar vinyl sulfonamides in the presence of excess $\mathrm{MeSO}_{2} \mathrm{Cl}$ and $\mathrm{NEt}_{3}$ in $\mathrm{DCM}$ at reflux, that allowed to avoid isolation of intermediate mesylate [13]. The reaction proceeded smoothly and gave model vinylsulfonamide 4 with $83 \%$ yield. The existence of the only set of signals in ${ }^{1} \mathrm{H}$ NMR spectrum of the obtained compound 4 and observed coupling constants of $15.8 \mathrm{~Hz}$, which belonged to vinyl protons, testified the formation of the most stable $E$-isomer of compound 4 (Scheme 2).<smiles>CN(C)S(=O)(=O)CC(O)c1ccc(Br)cc1</smiles><smiles>CNS(=O)(=O)/C=C/c1ccc(Br)cc1</smiles>

Scheme 2. Synthesis of model E-2-(4-bromophenyl)- $N, N$-dimethylethenesulfonamide 4.

Inspired by successful synthesis of compound 4, we tried to transfer applied conditions to the synthesis of vinyl derivative 1. In contrast, it turned out that the best choice for the synthesis of the target vinyl sulfone 1 was the use of higher excess of $\mathrm{MeSO}_{2} \mathrm{Cl}$ (2 eq.) and pyridine as solvent under heating. This difference may be attributed to the fact that elimination process for the molecule of compound 3 is influenced as by $\mathrm{SO}_{2} \mathrm{NMe}_{2}$-substituent, as by aromatic ring, and in the case of compound $\mathbf{5}$ only the effect of $\mathrm{SO}_{2} \mathrm{NMe}_{2}$-group is present (Scheme 3).<smiles>CC(O)CS(C)(=O)=O</smiles>

5

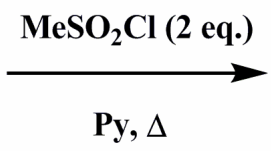

Py, $\Delta$<smiles>CC=CS(=O)(=O)N(C)C</smiles>

$1(43 \%)$

Scheme 3. Synthesis of the target $N, N$-dimethyl-1-propene-1-sulfonamide $\mathbf{1}$.

Compound 1 was isolated as a mixture of major $E$ - and minor $Z$-isomer. The assignment of the signals and the ratio between two isomers $(E: Z=88: 12)$ was established basing on their ${ }^{1} \mathrm{H}$ NMR-data. Namely, the coupling constant of $\mathrm{C}=\mathrm{CHSO}_{2}$-atom equals in the case of $E$-isomer to $15.1 \mathrm{~Hz}$, and for $Z$-isomer it equals to $11.2 \mathrm{~Hz}$.

Interestingly, that 1-(methylsulfonyl)-2-propanol 6, the simplest secondary $\beta$-hydroxysulfone and the initial substance for the synthesis of the target compound $\mathbf{2}$, was unknown to the moment. It was synthesized starting from 1-(methylsulfonyl)-2-propanone 7 by reduction with $\mathrm{NaBH}_{4}$ in $\mathrm{MeOH}$ (Scheme 4). 


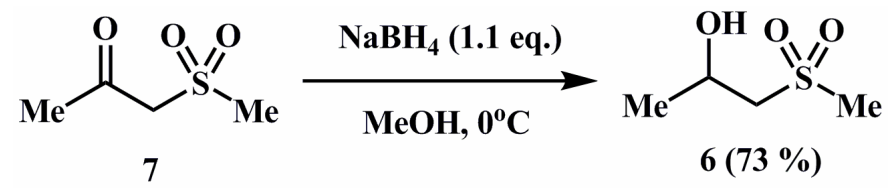

Scheme 4. Synthesis of 1-(methylsulfonyl)-2-propanol 6.

Expectedly, that compound 6, having more electron-withdrawing $\mathrm{MeSO}_{2}$-substituent (comparing with $\mathrm{SO}_{2} \mathrm{NMe}_{2}$-group in molecules of compounds 3 and $\mathbf{5}$ ) should form corresponding vinyl derivative under relatively milder conditions. Really, the formation of the target product $\mathbf{2}$ occurred in DCM under the action of 1.5 eq. of $\mathrm{MeSO}_{2} \mathrm{Cl}$ on compound $\mathbf{6}$ in the presence of 3 eq. of $\mathrm{NEt}_{3}$ at room temperature with good yield (Scheme 5).
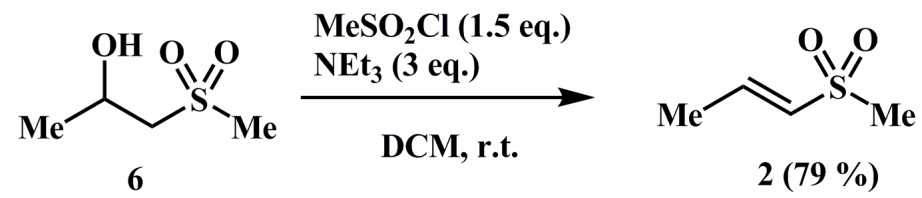

Scheme 5. Synthesis of the target $E$-1-(methylsulfonyl)-1-propene 2.

As well as for compound 4, formation of only $E$-isomer of the product $\mathbf{2}$ was detected using ${ }^{1} \mathrm{H}$ NMR spectrum, which showed the only $\mathrm{HC}=\mathrm{CHSO}_{2}$-coupling constant equal to $15.3 \mathrm{~Hz}$.

Synthesized compounds 1-4 are stable in time and in air for at least several months, that was also confirmed by the permanence of their spectral data.

\section{Conclusion}

Basing on the successful preliminary synthesis of E-2-(4-bromophenyl)$\mathrm{N}, \mathrm{N}$-dimethylethenesulfonamide, we worked up the simple and convenient protocols of the synthesis of (E/Z)-N,N-dimethyl-1-propene-1-sulfonamide and $E$-1-(methylsulfonyl)-1-propene, being, together with obtained 1-(methylsulfonyl)-2-propanol, among the most low molecular weight and little-known compounds in their series.

\section{Experimental}

${ }^{1} \mathrm{H}$ NMR spectra were registered in $\left(\mathrm{CD}_{3}\right)_{2} \mathrm{SO}\left(\delta_{\mathrm{H}}=2.50 \mathrm{ppm}\right)$ at $400 \mathrm{MHz}$ using Varian MR-400 spectrometer with $\mathrm{Si}\left(\mathrm{CH}_{3}\right)_{4}$ as an internal standard. Chemical shifts are given in ppm, coupling constants are given in Hz. Resonance multiplicity is described as s (singlet), doublet (d), m (multiplet) and br (broad signal). EI mass spectra were obtained with a Shimadzu GCMS-QP2020 instrument (70 eV ionizing energy) using direct inlet method. Argon of $99.993 \%$ purity was used for the syntheses with $n$-BuLi and $\mathrm{MeSO}_{2} \mathrm{Cl}$. Starting $n$ - $\mathrm{BuLi}\left(2.5 \mathrm{M}\right.$ in hexanes), $\mathrm{MeSO}_{2} \mathrm{Cl}$, 4-bromobenzaldehyde, triethylamine, inorganic reagents and solvents were commercially available. THF was dried over $\mathrm{KOH}$ and distilled over molten potassium before use. Dichloromethane (DCM) was dried over $\mathrm{K}_{2} \mathrm{CO}_{3}$. Triethylamine and pyridine were dried over KOH. N,N-Dimethylmethanesulfonamide and 2-hydroxy$\mathrm{N}, \mathrm{N}$-dimethyl-1-propanesulfonamide $\mathbf{5}$ were synthesized, as reported [12]. 1-(Methylsulfonyl)-2propanone 7 was obtained, as shown elsewhere [14, 15].

$N, N$-Dimethyl-1-propene-1-sulfonamide 1 , mixture of $E$ - and $Z$-isomers $(E: Z=88: 12)$. $\mathrm{MeSO}_{2} \mathrm{Cl}(27.5 \mathrm{~g}, 0.240 \mathrm{~mol})$ was added dropwise to a solution of 2-hydroxy- $N, N$-dimethyl1-propanesulfonamide $(20.0 \mathrm{~g}, 0.120 \mathrm{~mol})$ in anhydrous pyridine $(100 \mathrm{ml})$ at r.t. Resulting mixture was than heated for $48 \mathrm{~h}$. at $90^{\circ} \mathrm{C}$, cooled and poured into water $(800 \mathrm{ml})$ and extracted with $\mathrm{CHCl}_{3}(7 \times 40 \mathrm{ml})$. The obtained extract was washed with brine $(2 \times 40 \mathrm{ml})$, conc. $\mathrm{HCl}(3 \times 45 \mathrm{ml})$, brine $(40 \mathrm{ml})$, saturated aqueous $\mathrm{NaHCO}_{3}(2 \times 40 \mathrm{ml})$, brine $(2 \times 40 \mathrm{ml})$ and dried over $\mathrm{K}_{2} \mathrm{CO}_{3}$. After filtering the drying agent and evaporating the solvent $7.74 \mathrm{~g} \mathrm{(43 \% )}$ of the target product were obtained. Darkorange liquid. ${ }^{1} \mathrm{H} \mathrm{NMR}\left(\left(\mathrm{CD}_{3}\right)_{2} \mathrm{SO}\right), \delta$, ppm $(J, \mathrm{~Hz}): 6.54-6.65\left(1 \mathrm{H}, \mathrm{m}, E-\mathrm{CHCH}_{3}\right), 6.40-6.54(1 \mathrm{H}, \mathrm{m}$, $\left.Z-\mathrm{CHCH}_{3}\right), 6.43(1 \mathrm{H}, \mathrm{d}, J=15.1, E-\mathrm{C}=\mathrm{CHS}), 6.22(1 \mathrm{H}, \mathrm{d}, J=11.2, Z-\mathrm{C}=\mathrm{CHS}), 2.65(6 \mathrm{H}, \mathrm{s}$, $\left.Z-\mathrm{N}\left(\mathrm{CH}_{3}\right)_{2}\right), 2.60\left(3 \mathrm{H}, \mathrm{s}, E-\mathrm{N}\left(\mathrm{CH}_{3}\right)_{2}\right), 1.98\left(3 \mathrm{H}, \mathrm{d}, J=7.2, Z-\mathrm{CH} H_{3} \mathrm{CH}\right), 1.87(3 \mathrm{H}, \mathrm{d}, J=6.7$, $\left.E-\mathrm{CH}_{3} \mathrm{CH}\right) . \mathrm{m} / \mathrm{z}(\mathrm{EI}, 70 \mathrm{eV}): 149\left(\mathrm{M}^{+}, 27\right), 105$ (15), 44 (100), 41 (63). 
E-1-(Methylsulfonyl)-1-propene 2. $\mathrm{MeSO}_{2} \mathrm{Cl}(72.3 \mathrm{~g}, 0.631 \mathrm{~mol})$ was added dropwise to a solution of 1-(methylsulfonyl)-2-propanol $6(58.0 \mathrm{~g}, 0.420 \mathrm{~mol})$ and $\mathrm{NEt}_{3}(175 \mathrm{ml}, 1.26 \mathrm{~mol})$ in 11 of dry $\mathrm{DCM}$ at temperature below $0^{\circ} \mathrm{C}$. The resulting mixture was stirred for $72 \mathrm{~h}$. at r.t., washed with saturated aqueous $\mathrm{NaCl}(2 \times 300 \mathrm{ml})$, aqueous $10 \% \mathrm{HCl}(3 \times 200 \mathrm{ml})$, brine $(200 \mathrm{ml})$, saturated aqueous $\mathrm{NaHCO}_{3}(2 \times 200 \mathrm{ml})$, dried over $\mathrm{K}_{2} \mathrm{CO}_{3}$. After filtering the drying agent and evaporating the solvent $39.6 \mathrm{~g}(79 \%)$ of the product were obtained. Pale-yellow crystals after washing with mixture hexane/MTBE (2:1) and air drying. M.p. $38-9^{\circ} \mathrm{C} .{ }^{1} \mathrm{H}$ NMR $\left(\left(\mathrm{CD}_{3}\right)_{2} \mathrm{SO}\right), \delta, \quad p p m \quad(J, \mathrm{~Hz})$ : 6.68-6.77 $\left(1 \mathrm{H}, \mathrm{m}, \mathrm{CHCH}_{3}\right), 6.65(1 \mathrm{H}, \mathrm{d}, J=15.3, \mathrm{C}=\mathrm{CHS}), 2.93\left(3 \mathrm{H}, \mathrm{s}, \mathrm{SO}_{2} \mathrm{Me}\right), 1.86(3 \mathrm{H}, \mathrm{d}, J=5.5$, $\left.\mathrm{CH}_{3} \mathrm{CH}\right) . \mathrm{m} / \mathrm{z}(\mathrm{EI}, 70 \mathrm{eV}): 120\left(\mathrm{M}^{+}, 6\right), 105$ (17), 91 (28), 64 (22), 57 (37), 45 (53), 41 (100).

2-(4-Bromophenyl)-2-hydroxy- $N, \boldsymbol{N}$-dimethylethanesulfonamide 3. $n$-BuLi $(2.5 \mathrm{M}$ in hexanes, $27 \mathrm{ml}, 68 \mathrm{mmol})$ was added dropwise to a solution of $N, N$-dimethylmethanesulfonamide $(7.0 \mathrm{~g}$, $57 \mathrm{mmol}$ ) in $45 \mathrm{ml}$ of THF at temperatures below $-5^{\circ} \mathrm{C}$. The mixture was stirred under cooling for $1.5 \mathrm{~h}$ and a solution of 4-bromobenzaldehyde $(11.6 \mathrm{~g}, 63 \mathrm{mmol})$ in $20 \mathrm{ml}$ of THF was added dropwise at temperatures below $-5^{\circ} \mathrm{C}$. The resulting mixture was stirred for $12 \mathrm{~h}$. A conc. aqueous solution of $\mathrm{NH}_{4} \mathrm{Cl}(18 \mathrm{ml})$ and $750 \mathrm{ml}$ of water were added in turn. The mixture was extracted with EtOAc $(5 \times 100 \mathrm{ml})$, the extract was dried over $\mathrm{K}_{2} \mathrm{CO}_{3}$. After filtration of drying agent and evaporation of a solvent $16.5 \mathrm{~g}(94 \%)$ of the product were obtained. M. p. $76-8^{\circ} \mathrm{C} .{ }^{1} \mathrm{H}$ NMR $\left(\left(\mathrm{CD}_{3}\right)_{2} \mathrm{SO}\right), \delta$, ppm $(J$, $\mathrm{Hz}): 7.50(2 \mathrm{H}, \mathrm{d}, J=8.3, \mathrm{ArH}), 7.33(2 \mathrm{H}, \mathrm{d}, J=8.3, \mathrm{ArH}), 5.83(1 \mathrm{H}$, br. s, OH), $4.93(1 \mathrm{H}, \mathrm{dd}, J=8.7$, $J=3.5, \mathrm{C} H \mathrm{OH}), 3.35\left(1 \mathrm{H}, \mathrm{dd}, J=14.5, J=8.7, \mathrm{CH}_{A} \mathrm{H}_{\mathrm{B}}\right), 3.17\left(1 \mathrm{H}, \mathrm{dd}, J=14.5, J=3.5, \mathrm{CH}_{\mathrm{A}} H_{B}\right)$, $2.72\left(6 \mathrm{H}, \mathrm{s}, \mathrm{N}\left(\mathrm{CH}_{3}\right)_{2}\right) . \mathrm{m} / \mathrm{z}(\mathrm{EI}, 70 \mathrm{eV}): 308\left(\mathrm{M}^{+}, 1\right), 198$ (80), 185 (57), 120 (36), 92 (67), 77 (48), 44 (100).

E-2-(4-Bromophenyl)- $\boldsymbol{N}, \boldsymbol{N}$-dimethylethenesulfonamide $4 . \mathrm{MeSO}_{2} \mathrm{Cl}(50.8 \mathrm{~g}, 0.44 \mathrm{~mol})$ was added dropwise to a solution of 2-(4-bromophenyl)-2-hydroxy- $N, N$-dimethylethanesulfonamide 3 $(80.2 \mathrm{~g}, 0.26 \mathrm{~mol})$ and $\mathrm{NEt}_{3}(126 \mathrm{ml}, 0.91 \mathrm{~mol})$ in $710 \mathrm{ml}$ of dry DCM at temperatures below $0^{\circ} \mathrm{C}$. The resulting mixture was heated to reflux for $24 \mathrm{~h}$., cooled, washed with water $(3 \times 400 \mathrm{ml})$, aqueous $20 \%$ $\mathrm{HCl}(3 \times 60 \mathrm{ml})$, saturated aqueous $\mathrm{NaHCO}_{3}(4 \times 100 \mathrm{ml})$, water $(400 \mathrm{ml})$, dried over $\mathrm{K}_{2} \mathrm{CO}_{3}$. After filtering the drying agent and evaporating the solvent $63.0 \mathrm{~g}(83 \%)$ of the product were obtained. Paleyellow crystals. M. p. $128-9^{\circ} \mathrm{C}(\mathrm{EtOAc}) .{ }^{1} \mathrm{H}$ NMR $\left(\left(\mathrm{CD}_{3}\right)_{2} \mathrm{SO}\right), \delta$, ppm $(J, \mathrm{~Hz}): 7.70(2 \mathrm{H}, \mathrm{d}, J=8.0$, $\mathrm{ArH}), 7.61(2 \mathrm{H}, \mathrm{d}, J=8.0, \mathrm{ArH}), 7.36(1 \mathrm{H}, \mathrm{d}, J=15.8, \mathrm{C}=\mathrm{CHAr}), 7.31(1 \mathrm{H}, \mathrm{d}, J=15.8, \mathrm{SCH}=\mathrm{C})$, $2.69\left(6 \mathrm{H}, \mathrm{s}, \mathrm{N}\left(\mathrm{CH}_{3}\right)_{2}\right) . \mathrm{m} / \mathrm{z}(\mathrm{EI}, 70 \mathrm{eV}): 291\left(\mathrm{M}\left({ }^{81} \mathrm{Br}\right)^{+}, 21\right), 289\left(\mathrm{M}\left({ }^{79} \mathrm{Br}\right)^{+}, 20\right), 225(70), 181(31)$, $102(100), 75(20), 44(23)$.

1-(Methylsulfonyl)-2-propanol 6. $\mathrm{NaBH}_{4}(30.8 \mathrm{~g}, 0.81 \mathrm{~mol})$ was added portionwise to a solution of 1-(methylsulfonyl)-2-propanone 7 (100 g, $0.73 \mathrm{~mol})$ in $\mathrm{MeOH}(800 \mathrm{ml})$ under bubbler at temperatures below $0^{\circ} \mathrm{C}$. The resulting mixture was stirred for 24 h. at r.t., the solvent was removed under reduced pressure. $\mathrm{CHCl}_{3}(600 \mathrm{ml})$ and saturated aqueous $\mathrm{Na}_{2} \mathrm{CO}_{3}(150 \mathrm{ml})$ were added to the residue and the resulting mixture was allowed to stir for $1.5 \mathrm{~h}$. Organic phase was collected, dried over $\mathrm{K}_{2} \mathrm{CO}_{3}$. After filtering the drying agent and evaporating the solvent $74.5 \mathrm{~g}(73 \%)$ of the product were obtained. Pale-yellow crystals. M. p. $61-3^{\circ} \mathrm{C}{ }^{1} \mathrm{H}$ NMR $\left(\left(\mathrm{CD}_{3}\right)_{2} \mathrm{SO}\right), \delta$, ppm $(J, \mathrm{~Hz}): 5.10(1 \mathrm{H}, \mathrm{br} . \mathrm{s}, \mathrm{OH})$, 4.01-4.13 (1H, m, CH), $3.19\left(1 \mathrm{H}, \mathrm{dd}, J=14.6, J=8.6, \mathrm{CH}_{A} \mathrm{H}_{\mathrm{B}}\right), 3.00(1 \mathrm{H}, \mathrm{dd}, J=14.6, J=3.0$, $\left.\mathrm{CH}_{\mathrm{A}} H_{B}\right), 2.94\left(3 \mathrm{H}, \mathrm{s}, \mathrm{SO}_{2} \mathrm{CH}_{3}\right), 1.14\left(3 \mathrm{H}, \mathrm{d}, J=6.3, \mathrm{CH}_{3} \mathrm{CH}\right) . \mathrm{m} / \mathrm{z}(\mathrm{EI}, 70 \mathrm{eV}): 139$ (52), 123 (66), 121 (48), 94 (100), 79 (66), 59 (41), 45 (33), 41 (43), 31 (56), 15 (32).

\section{Acknowledgment}

Authors thank V. I. Musatov ("Institute for Single Crystals" NAS of Ukraine, Kharkiv, Ukraine) for spectroscopic measurements.

\section{References}

1. Fang Y., Luo Z., Xu X. Recent advances in the synthesis of vinyl sulfones. RSC Adv. 2016, 6, 59661-59676. https://doi.org/10.1039/C6RA10731A.

2. Craven G. B., Affron D. P., Raymond P. N., Mann D. J., Armstrong A. Vinyl sulfonamide synthesis for irreversible tethering via a novel $\alpha$-selenoether protection strategy. Med. Chem. Commun. 2019, 10, 158-163. https://doi.org/10.1039/C8MD00566D.

3. Meadows D. C., Gervay-Hague J. Vinyl sulfones: synthetic preparations and medicinal chemistry applications. Med. Res. Rev. 2006, 26, 793-814. https://doi.org/10.1002/med.20074. 
4. Sulzer-Mossé S., Alexakis A., Mareda J., Bollot G., Bernardinelli G., Filinchuk Ya. Enantioselective Organocatalytic Conjugate Addition of Aldehydes to Vinyl Sulfones and Vinyl Phosphonates as Challenging Michael Acceptors. Chem Eur. J. 2009, 15, 3204-3220. https://doi.org/10.1002/chem.200801892.

5. Mulet C., Escolano M., Llopis S., Sanz S., de Arellano C. R., Sánchez-Roselló M., Fustero S., del Pozo C. Dual Role of Vinyl Sulfonamides as $N$-Nucleophiles and Michael Acceptors in the Enantioselective Synthesis of Bicyclic $\delta$-Sultams. Adv. Synth. Catal., 2018, 360, 2885-2893. https://doi.org/10.1002/adsc.201800548.

6. Rogachev V. O., Metz P. Thermal and high pressure intramolecular Diels-Alder reaction of vinylsulfonamides. Nat. Protoc. 2006, 1, 3076-3087. https://doi.org/10.1038/nprot.2006.463.

7. Philips J. C., Oku M. Preparation of the Diels-Alder adducts of methyl vinyl sulfone and cyclopentadiene and of their dihydro derivatives. J. Org. Chem. 1972, 37, 26, 4479-4480. https://doi.org/10.1021/jo00799a048.

8. Helwig D., Pritzkow W., Radeglia R., Schmidt-Renner W., Ziegler J. Untersuchungen zur Sulfochlorierung von Paraffinen. VI. Untersuchungen über die Sulfochlorierung von definierten Alkylchloriden. J. Prakt. Chem. 1980, 322, 281-90. https://doi.org/10.1002/prac.19803220214.

9. Liu L. K., Hwang W. S. Syntheses and mass spectral studies of $(E)-1$-alkanesulfonylpropenes and (E)-1-alkanesulfinylpropenes. J. Chin. Chem. Soc. 1984, 31, 357-367. https://doi.org/10.1002/jccs.198400051.

10. O'Connor D. E., Lyness W. I. The effect of methylmercapto, methylsulfinyl, and methylsulfonyl groups on the equilibrium in three-carbon prototropic systems. J. Am. Chem. Soc. 1964, 86, 3840-3846. https://doi.org/10.1021/ja01072a048.

11. Kolosov M. A., Al-Ogaili M. J. K., Kulyk O. G., Orlov V. D. Synthesis of 4,7-dihydro[1,2,4]triazolo[1,5-a]pyrimidine-6-sulfonamide derivatives. Chem. Heterocycl. Compd. 2015, 51, 691-694. https://doi.org/10.1007/s10593-015-1759-5.

12. Shvets E. H., Pidvorotnia A. V., Kulyk O. G., Mazepa A. V., Kolosov M. A. A straightforward synthesis of 5-sulfonamidomethyl substituted 4,7-dihydroazolo[1,5-a]pyrimidines. Synth. Commun. 2021, 51, 114-122. https://doi.org/10.1080/00397911.2020.1821224.

13. Thompson M. E. $\alpha, N$-Alkanesulfonamide Dianions: Formation and Chemoselective C-Alkylation. J .Org. Chem. 1984, 49, 1700-1703. https://doi.org/10.1021/jo00184a006.

14. Gladkov E. S., Chebanov V. A., Desenko S. M., Shishkin O. V., Shishkina S. V., Dallinger D., Kappe C. O. Multicomponent cyclocondensations of $\beta$-ketosulfones with aldehydes and aminoazole building blocks. Heterocycles 2007, 63, 469-480. https://doi.org/10.3987/COM-07-S(U)19.

15. 15. Safrygin A., Dar'in D., Kantin G., Krasavin M. $\alpha$-Diazo- $\beta$-oxosulfones as Partners in the Wolff 1,2,3-Triazole Synthesis and the Wolff Rearrangement in the Presence of Aromatic Amines. Eur. J. Org. Chem. 2019, 29, 4721-4724. https://doi.org/10.1002/ejoc.201900698.

Надіслано до редакиії 15 жовтня 2020 р.

І.Д. Гужва, О.Г. Швець, М.О. Колосов. Синтез 1-(метилсульфоніл)-1-пропену та N,N-диметил-1-пропен1-сульфонаміду.

Харківський національний університет імені В.Н. Каразіна, хімічний фракультет, майдан Свободи, 4, Харків, 61022, Україна

Різноманітні вінілсульфони та вінілсульфонаміди мають широкий спектр біологічної активності (це, в основному, інгібування різних типів ферментів) і часто використовуються в синтетичній органічної хімії (як активні діенофіли, акцептори Міхаеля і, в цілому, активні агенти в реакціях 1,4-приєднання та електроціклізаціі). Однак, незважаючи на популярність великої кількості подібних сполук, препаративні методики одержання низькомолекулярних представників цього класу - 1-(метилсульфоніл)-1-пропену і $N, N$-диметил1-пропен-1-сульфонаміду - до теперішнього часу вивчені мало. У цій роботі ми повідомляємо про простий, ефективний і загальний спосіб синтезу 1-(метилсульфоніл)-1-пропену і $N, N$-диметил-1-пропен1-сульфонаміду шляхом дегідратації, відповідно, 1-(метилсульфоніл)-2-пропанолу та $N, N$-диметил2-гідроксіпропансульфонаміду в системі $\mathrm{MeSO}_{2} \mathrm{Cl} /$ органічна основа, спираючись на попередній експеримент з синтезу 2-(4-бромфеніл)- N,N-диметилвінілсульфонаміду виходячи 3 2-(4-бромфеніл)-2-гідрокси$N, N$-диметилетансульфонаміду. Останній, у свою чергу, було отримано, виходячи 3 $N, N$-диметилметансульфонаміду шляхом літіювання за допомогою $\mathrm{H}$-BuLi, подальшої дії 
4-бромбензальдегіду і наступної обробки. Використана методика синтезу вінілпохідних дозволяє уникнути виділення проміжних мезилатів і полягає в однореакторному формуванні відхідної групи та її відщеплення. Згідно констант спін-спінового розщеплення у спектрах ${ }^{1} \mathrm{H}$ ЯМР, отриманий $N, N$-диметил-1-пропен1-сульфонамід існує у вигляді суміші E- і Z-ізомерів (у співвідношенні 88:12), у той час як виділені 1-(метилсульфоніл)-1-пропен і 2-(4-бромфеніл)- $N, N$-диметилвінілсульфонамід $\epsilon$ найбільш стабільними Е-изомерами. Будову синтезованих сполук підтверджено методами ${ }^{1} \mathrm{H}$ ЯМР-спектроскопії та масспектрометрії.

Ключові слова: вінілсульфони, вінілсульфонаміди, 1-(метилсульфоніл)-1-пропен, $N, N$-диметил1-пропен-1-сульфонамід, дегідратація.

И.Д.Гужва, Е.Г.Швец, М.А.Колосов. Синтез 1-(метилсульфонил)-1-пропена и N,N-диметил-1-пропен1-сульфонамида.

Харьковский национальный университет имени В.Н. Каразина, химический фракультет, пл. Свободы, 4 , Харьков, 61022, Украина

Различные винилсульфоны и винилсульфонамиды обладают широким спектром биологической активности (это, в основном, ингибирование различных типов ферментов) и часто используются в синтетической органической химии (в качестве активных диенофилов, акцепторов Михаэля и, в целом, активных агентов в реакциях 1,4-присоединения и электроциклизации). Однако, несмотря на известность большого количества подобных соединений, препаративные методики получения низкомолекулярных представителей этого класса - 1-(метилсульфонил)-1-пропена и N,N-диметил-1-пропен-1-сульфонамида - до настоящего времени изучены мало. В настоящей работе мы сообщаем о простом, эффективном и общем способе синтеза 1-(метилсульфонил)-1-пропена и $N, N$-диметил-1-пропен-1-сульфонамида путём дегидратации, соответственно, 1-(метилсульфонил)-2-пропанола и N,N-диметил-2-гидроксипропансульфонамида в системе $\mathrm{MeSO}_{2} \mathrm{Cl} /$ органическое основание, опираясь на предварительный эксперимент по синтезу 2-(4-бромфенил)- $N, N$-диметилвинилсульфонамида исходя из 2-(4-бромфенил)-2-гидрокси$N, N$-диметилэтансульфонамида. Последний, в свою очередь, был получен исходя из $N, N$-диметилметансульфонамида путем литирования с помощью H-BuLi, последующего действия 4-бромбензальдегида и дальнейшей обработки. Использованная методика синтеза винилпроизводных позволяет избежать выделения промежуточных мезилатов и состоит из однореакторного формирования уходящей группы и ее отщепления. Согласно константам спин-спинового расщепления в спектрах ${ }^{1} \mathrm{H}$ ЯМР, полученный $N, N$-диметил-1-пропен-1-сульфонамид существует в виде смеси E- и Z-изомеров (в соотношении 88:12), В то время как выделенные 1-(метилсульфонил)-1-пропен и 2-(4-бромфенил)$N, N$-диметилвинилсульфонамид являются наиболее стабильными E-изомерами. Строение синтезированных соединений подтверждено методами ${ }^{1} \mathrm{H}$ ЯМР-спектроскопии и масс-спектрометрии.

Ключевые слова: винилсульфоны, винилсульфонамиды, 1-(метилсульфонил)-1-пропен, N,N-диметил1-пропен-1-сульфонамид, дегидратация.

Kharkiv University Bulletin. Chemical Series. Issue 35 (58), 2020 